Downstream Impact of an Active-Learning-Based Engineering Physics - Mechanics Course

Dr. Timothy J. Garrison, York College of Pennsylvania

Timothy Garrison is Chair of the Engineering and Computer Science Department 


\title{
Downstream Impact of an Active-Learning-Based Engineering Physics - Mechanics Course
}

\begin{abstract}
At the 2014 and 2015 Annual ASEE conferences, the author presented papers on a completely restructured engineering physics - mechanics course. The traditional physics course structure, consisting of a separate lecture ( $3 \mathrm{hrs}$ three times per week), laboratory ( $3 \mathrm{hrs}$ once a week) and recitation (1.5 hours once a week), was discarded in favor of a single, blended class meeting 2.5 hours three times per week. Moreover, the new class was designed to operate as a fully active learning course (i.e. without any lecture) by making use of several active learning methods including peer instruction (aka think-pair-share) and interactive, peer laboratories. The restructured course was assessed using the Force Concept Inventory (FCI) assessment test, given on the first and last days of class. Results from the FCI test show that the overall gain in performance increased from $12 \%$ to $33 \%$ as a result of the combined effects of these changes. Additionally, the overall pass rate for the course (grade of " $\mathrm{C}$ " or better) increased from $53 \%$ to $75 \%$. While these results are very positive, it is productive to explore the longer-term effects of this restructuring. In particular, did the students taking the restructured course do better in the follow-on courses (E\&M Physics, Statics, and Dynamics) than the students who took the course in its original format? Alternately, does the increased gain on the FCI test result in better conceptual understanding that carries through to future uses of the material? The author has collected data to explore this by tracking the performance of five year's worth of students (a total of about 400 students) that took the course in the restructured format as compared to a similar number of students that took the course in the original format. The performance of those two groups of students in follow-on courses has been analyzed and shows improved performance (overall grades and pass rates) in the follow-on courses.
\end{abstract}

\subsection{Introduction}

In the sciences, the overall structure for educating students has remained nearly unchanged over the years. Be it a biology, chemistry, or physics class, students typically learn by attending a classroom several times per week and participating in a longer, weekly group laboratory. Frequently the classroom experience is comprised of lectures and it is fairly common for the laboratory and lecture to be administered by different individuals.

While this structure may be effective at processing students through the science courses, studies have shown that it has some significant educational disadvantages. Over the past several decades, physics education research has shown that students were not learning the concepts and/or were not engaged by the methods used in "traditional" physics education. ${ }^{1-4}$ Those and other studies have motivated a significant amount of research on physics education and much progress has been made. A significant body of physics education research has focused on developing and incorporating classroom techniques that reduce or eliminate lecture and replace it with active learning methods. ${ }^{5,6}$ Often the focus of the active learning strategies has been to move away from methods that lead to students memorizing facts and mimicking solutions and 
toward developing conceptual knowledge. ${ }^{7}$ Other studies have looked at changing/enhancing the experimental/laboratory component. ${ }^{6,8}$

Physics education research has also focused on developing quantitative methods that can be used to assess the effectiveness of the traditional teaching structure as well as the impact of new teaching strategies. Those efforts led to the development of a number of standardized physics assessment tests. ${ }^{9,10}$ Via administration of the assessment tests, numerous physics educators have shown that active learning methods and improved laboratory experiences provide substantial gains over the traditional lecture/lab format. Details of these methods, their assessment, and the evolution of physics education research have been documented in several books on physics education strategies. $^{5,11,12}$

Despite the fact that the traditional structure has been shown to be relatively ineffective and that a number of new techniques have been shown to significantly increase conceptual understanding, many courses continue to use the traditional lecture/lab format or have only incorporated a few select techniques. Beginning in 2008, the author worked to transform a traditional physics course (with separate lecture, laboratory, and recitation components) into a blended structure where all of the components are amalgamated together. Additionally, the lecture elements have been substantially reduced in favor of active learning techniques. The transformation has been conducted in phases that included: blending the separate lecture, laboratory and recitation elements into a combined experience; development of interactive laboratories; introduction of electronic response systems (clickers); incorporation of peer instruction (a.k.a. think-pair-share) questions; and, development of an active learning textbook ${ }^{13}$. Each year, as new features were phased in, pre- and post-course assessments were conducted using the Force Concept Inventory (FCI) test. ${ }^{9}$ The FCI test results showed substantial performance gains as each of the phases were implemented. With all of the changes that have been incorporated thus far, the gain in the FCI test is approximately triple that of the traditional lecture and laboratory format. Details on the evolution of the class, the active learning methods that were used, results of the annual assessment tests, and best practices/advice for each of the methods were presented and published at the 2014 and 2015 ASEE Annual Conferences. ${ }^{14,15}$

While the concept inventory tests are very valuable for assessing gains within a course, they do not give any information on the lasting effects of those gains. In the author's situation, substantial changes to the physics-mechanics class resulted in significant gains in the FCI test scores. Those gains indicate an improved understanding of conceptual knowledge from start to end of that particular course. What is not clear is how lasting those gains are. That is, do the gains in conceptual understanding carry through to subsequent courses that make use of the same concepts?

This paper focuses on assessing the downstream impact of the changes made to the engineering physics-mechanics course. This was done by looking at student performance in three courses that have direct conceptual connection to the mechanics course: 1) engineering physics electricity and magnetism; 2) statics; and 3) dynamics. 
Included in the following sections is a more in-depth review of the changes that were made to the engineering physics-mechanics course, a summary of the conceptual gains achieved in that course, and an assessment of student performance gains in the three related courses noted above.

\subsection{Background}

\subsection{Motivation}

Up through the 2008 offerings, the Engineering Physics - Mechanics course was taught in the traditional style. It was five credit hours and consisted of a three-credit lecture, a one-credit laboratory, and a one-credit recitation. In total, these accounted for seven and a half hours of contact with the students per week.

A careful review of the course revealed a number of problems including ${ }^{13}$ :

- High failure rates

- Low comprehension rates in lecture

- Marginal contribution from the laboratories

- Disconnect between lecture and labs

Starting in 2009, the separate lecture, recitation, and lab components were abandoned in favor of a blended class that incorporates all of those elements. The new course structure was designed to meet three times per week, with each meeting being two and a half hours long. The net meeting time with the students remained unchanged at 7.5 hours per week and the course remained five credit hours.

\subsection{New Structure}

To implement the new course structure, a special classroom was built to facilitate active learning and to stimulate student interactions. Students sit in groups of three at lab tables. The tables are configured such that any group of students can interact with several other groups. (The classroom was designed to hold 45 students.)

Because of the active nature of the new course structure, there is a need for considerable interaction between the students and instructor. To facilitate those interactions, each two-and-ahalf-hour class is staffed by two instructors. At the author's institution either two full-time faculty members or a full-time faculty member and an adjunct faculty staff the course. (York College does not offer graduate programs in engineering and does not use teaching assistants.) When the students are engaged in active learning, which is a majority of the time, both instructors float among the class and interact with the students. Currently, three sections are offered in the Spring term and one in the Fall term.

To incorporate active learning within the classroom, several mechanisms are used. Those methods include peer instruction (aka think-pair-share), interactive labs, electronic clickers, and online lecture videos. 
In the peer instruction process, students are presented with a question/task that addresses a conceptual topic. After presenting the students with the question, they are given a brief period to think about it and respond. This can take many forms such as making a predictive graph, drawing a diagram, performing an analysis, or answering a conceptual multiple-choice question via an electronic clicker. The students initially respond individually. After submitting their own responses, the students are allowed to discuss the question with their group mates and, if they like, with other groups and/or one of the instructors. After the group discussions, the students address the same exercise again. Finally, if necessary, one of the instructors discusses the question to clear up any misconceptions.

In the interactive laboratories, the instructor controls a single, pre-configured laboratory setup at the front of the room. In a typical interactive laboratory experience the instructor reviews the experimental setup with the students and poses a question or task. Next, each student makes a self-prediction for the outcome followed by a group prediction. The experiment is then conducted and the results are discussed. It is important to note that the interactive laboratories are not simply demonstrations that the students passively observe. During a typical interactive laboratory the students: provide input on the experiment, the equipment used and its setup; do pre-analysis to develop the supporting theory; provide input on how to collect and process the data; perform analysis of the results; discuss sources of error; and suggest potential improvements. Details on the interactive laboratories are included in reference 15.

As noted above, to facilitate rapid feedback from the students during class, electronic response units ("clickers") are used. The data collected from these units have proven extremely valuable for assessing student comprehension, for identifying where additional discussion is needed, and for pacing the class.

\subsection{Course-Level Assessment}

One of the primary quantitative assessment methods used to assess the benefits of the new course structure was the Force Concept Inventory (FCI) test ${ }^{9}$. The FCI assessment test was administered each year (pre- and post-class) over a span of six years. The FCI test consists of 30 multiplechoice questions that examine conceptual knowledge of mechanics (i.e. there are no quantitative questions). According to Hake ${ }^{16}$ who has studied application of the FCI test in over six thousand students, the best metric for presenting the results is the normalized gain given by

$$
G=\left[\frac{(\text { post class average })-(\text { pre class average })}{100-(\text { pre class average })}\right] * 100 \%
$$

Student grades and course surveys were also used to assess the course changes. Complete details can be found in reference 15 . The following section summarizes the results from the FCI test and presents select grade data.

Table 1 gives the results from the FCI test over a seven-year period. The table gives the number of students enrolled, N, the pre- and post-class averages on the FCI test, the average FCI gain calculated using equation (1), and the pass rate for the course. 
Data for 2008 represent the last time the course was offered in the original lecture/lab format described in section 2.1. It is important to note that the FCI post-test was not administered in 2008. The gain of 11.7 was estimated using information compiled by Hake for lecture-based physics classes. The pass rate for the course was 53.2\% which was typical for offerings prior to 2008 (using the traditional lecture/lab format). In calculating the pass rate, grades of D, F or W (withdrawal) were considered failures.

Table 1: FCI and Pass Rate Results

\begin{tabular}{|c|c|c|c|c|c|c|}
\hline Year & N & Pre & Post & G & Pass Rate & Phase* $^{* 1}$ \\
\hline 2008 & 80 & 49 & - & 11.7 & 53.2 & 0 \\
\hline 2009 & 85 & 47 & 65 & 18 & 53.4 & 1 \\
\hline 2010 & 71 & 49 & 78 & 29 & 63.9 & 2 \\
\hline 2011 & 74 & 50 & 83 & 33 & 66.2 & 3 \\
\hline 2012 & 86 & 51 & 83.5 & 32.5 & 67.0 & 4 \\
\hline 2013 & 78 & 48.5 & 81.5 & 33 & 59.0 & 4 \\
\hline 2014 & 81 & 48.9 & 80.1 & 31.2 & 75.0 & 4 \\
\hline
\end{tabular}

*The Phase refers to the structure of the course, as delineated below:

- Phase 0 - the course was offered in the "traditional" lecture/lab format

- Phase 1 - the first offering of the course with the lecture, laboratory, and recitation blended together. A modest amount of active learning was introduced via a reduced suite of interactive laboratory demonstrations and classroom exercises.

- Phase 2 - The suite of interactive laboratories was expanded significantly and numerous revisions were made to the activities. Overall, a substantial amount of active learning was added.

- Phase 3 - Peer instruction and electronic response units (clickers) were included. The first version of the active learning workbook ${ }^{13}$ was introduced. The interactive laboratories where further revised, broken into smaller segments, and distributed more evenly throughout the course to better mesh them with the lecture content.

- Phase 4-Modest revisions to the interactive laboratories, peer instruction questions, and the active learning workbook were implemented.

As shown in Table 1, the initial blending of the course and the introduction of a reduced set of interactive laboratories (Phase 1) boosted the gain to 18. This result falls within the bounds of other Interactive Engagement courses discussed by Hake, for which the range in gain would be 15.9 to 37.1. Given that only a limited amount of active learning was incorporated in this phase of the course's development, it makes sense that the gain would be closer to the lower limit.

Table 1 show that continued efforts to phase in more active learning resulted in significant improvements in both the FCI gain, G, and the pass rate of the course. According to Hake, the upper limit on gain for students whose pre-test score was $50 \%$ is $G_{\max }=35$. The gains from 2011 and beyond are all around 33 and are approaching the upper limit of the gains reported by Hake. Additionally, over the last three years the pass rate for the course has averaged $67 \%$ compared to an average of 53.3\% for the four-year span from 2005-2008 using the traditional structure. This represents a growth in pass rate of over $13 \%$. 
These results show the switch to the new physics course structure (and the associated implementation of active learning methods) have proven to be very beneficial at improving conceptual understanding. However, these gains were all assessed within the engineering physics - mechanics course via administration of the FCI on the first and last days of class. The author was also interested in learning if these gains in conceptual understanding carry forward to additional classes that rely heavily on the concepts covered in the physics-mechanics course. This is discussed in the following sections.

\subsection{Assessment of Follow-on Courses}

Since there is no physics major at the author's institution, York College of Pennsylvania, the vast majority of the students served by the engineering physics courses are engineering and engineering management majors. For the engineering students, there are three courses that follow from the mechanics physics course and make use of the conceptual knowledge conveyed in the class. They are engineering physics - electricity and magnetism (taken by all engineering majors), statics (taken by mechanical engineering and engineering management majors), and dynamics (taken by mechanical engineering majors). The author set out to assess if there were any performance gains in these follow-on courses that aligned with the implementation of the new physics-mechanics course.

While the FCI assessment is a very powerful tool for assessing conceptual understanding gained from an individual course, the author did not have a similar instrument to assess the downstream impact in future courses. One reason for this was simply that the author had not thought to administer a downstream assessment when the changes to the physics - mechanics course were implemented. However, since nearly all assessments of learning are done at the course level, it is doubtful that any concept inventories exist for assessing a longer chain of courses. (A literature search did not reveal any.) Thus, the only mechanism that was available to explore a link was student grade data in the follow-on classes.

\subsection{Results}

\subsection{Engineering Physics-Mechanics Course}

Before considering the follow-on courses, it is useful to consider the grade data for the engineering physics-mechanics course. Referring to Table 1, there was a five-year period from 2010 to 2014 for which the nature of the new course was fairly similar (phases 2 to 4 ). In all of these phases the course was blended together and made heavy use of active learning. During that five-year span, the course was taught exclusively by the author. The author also considered the five-year period prior to 2010, for which the course was taught in the traditional lecture/lab/recitation format. (In 2009, the course elements were combined but only modest amounts of active learning were added). Table 2 summarizes the grade data over the complete ten-year period. The table includes the number of students in the course each year, the average GPA for the class (neglecting withdrawals), the percentage of the students that passed the course (requires a grade of "C" or better), and the percentage of the students that earned a "B" or better. The years shaded in green were taught using the new course structure with active learning. 
Table 2: Grade Data for Engineering Physics-Mechanics Course

\begin{tabular}{|c|c|c|c|c|}
\hline Year & N & Ave GPA & Pass Rate & \% “B” or higher \\
\hline 2005 & 54 & 2.14 & 57.4 & 38.6 \\
\hline 2006 & 46 & 1.86 & 50.0 & 33.3 \\
\hline 2007 & 59 & 2.06 & 52.5 & 38.0 \\
\hline 2008 & 79 & 2.26 & 53.2 & 41.3 \\
\hline 2009 & 73 & 1.97 & 53.4 & 31.0 \\
\hline 2010 & 72 & 2.19 & 63.9 & 37.7 \\
\hline 2011 & 74 & 2.09 & 66.2 & 36.6 \\
\hline 2012 & 91 & 2.34 & 67.0 & 35.1 \\
\hline 2013 & 78 & 2.29 & 59.0 & 41.0 \\
\hline 2014 & 80 & 2.38 & 75.0 & 47.4 \\
\hline
\end{tabular}

Table 3 shows the average results for the 2005 - 2009 offerings compared to the average results from $2010-2014$.

Table 3: Five-Year Before and After Averages

\begin{tabular}{|c|c|c|c|c|}
\hline Years & N & Ave GPA & Pass Rate & \% “B” or higher \\
\hline $2005-2009$ & 311 & 2.06 & 53.3 & 36.45 \\
\hline $2010-2014$ & 395 & 2.26 & 66.2 & 39.56 \\
\hline
\end{tabular}

Table 3 shows that the overall GPA, pass rate, and percentage of students earning a grade of "B" or better have all increased under the new course format. This is consistent with the increased gains in the FCI test that occurred with the restructured course (see Table 1) and supports the premise that increased conceptual understanding (as measured by the FCI) translates into improved problem-solving skills (as measured by the course exams and homework assignments).

As noted earlier, the mechanics course directly feeds into three courses, engineering physic E\&M, statics, and dynamics. A similar analysis to that shown in Tables 2 and 3 was done for each of these courses. For each of the follow-on courses, five year's worth of students who entered the course having taken the old version of the physics-mechanics class were averaged and compared against five year's worth of students who entered the class having taken the new version. The results are discussed in the following sections.

\subsection{Engineering Physics - Electricity \& Magnetism Course}

Table 4 shows averaged grade data for the electricity and magnetism class. The results show a significant increase in the average course GPA, the course pass rate, and the percentage of students earning a "B" or better. For reference, at the author's institution the students take the physics-mechanics course in the spring of their freshman year. The E\&M class is taken in the fall of their sophomore year (i.e. approximately 3 months after completing the mechanics course). 
Table 4: Five-Year Before and After Averages for Physics - E\&M

\begin{tabular}{|c|c|c|c|c|}
\hline Years & N & Ave GPA & Pass Rate & \% “B” or higher \\
\hline $2005-2009$ & 222 & 2.41 & 74.9 & 46.7 \\
\hline $2010-2014$ & 353 & 2.85 & 87.9 & 62.5 \\
\hline
\end{tabular}

\subsection{Statics Course}

Table 5 shows a similar dataset for the statics course. This course is taken by the mechanical engineering and engineering management students, with the engineering management students making up a small fraction of the total enrollment. Like the E\&M course, the statics course is taken in the fall semester of the sophomore year.

Table 5: Five-Year Before and After Averages for Statics

\begin{tabular}{|c|c|c|c|c|}
\hline Years & N & Ave GPA & Pass Rate & \% “B” or higher \\
\hline $2005-2009$ & 164 & 2.34 & 72.6 & 46.1 \\
\hline $2010-2014$ & 248 & 2.45 & 78.9 & 47.2 \\
\hline
\end{tabular}

As with the E\&M course, all of the metrics in Table 5 increased for students who entered the statics class having taken the restructured physics-mechanics course, albeit by smaller amounts. As noted in a later section, using grade data to assess improvements has many limitations, one of which is that not all instructors grade the same way. The data for the statics class provided an opportunity to remove some of that variation. In 2008 and 2009, the author taught both the statics class and the physics-mechanics class. For those two years, the physics was taught in the old format (2008) and in a very early version of the new format (2009). Likewise, the author taught both the statics class and physics-mechanics courses in 2011 and 2012, for which the physics was taught using the new structure. Table 6 shows the grade comparisons for both of those two-year periods.

Table 6: Two-Year Before and After Averages for Statics - Same Instructor

\begin{tabular}{|c|c|c|c|c|}
\hline Years & N & Ave GPA & Pass Rate & \% “B” or higher \\
\hline $2008-2009$ & 80 & 2.31 & 65.1 & 47.7 \\
\hline $2011-2012$ & 109 & 2.43 & 81.8 & 44.4 \\
\hline
\end{tabular}

\subsection{Dynamics Course}

The results for the dynamics class are shown in Table 7. Dynamics is only taken by mechanical engineering students and it is taken in their junior year. For 2006 through 2014, dynamics was offered in the fall term. For subsequent years it has been offered in the summer term at the end of the junior year. (The author's institution requires all engineering students to complete three semesters of full-time cooperative work experience. As a result, the engineering students take a full suite of classes in the summer of their junior and senior years.) Students taking dynamics have already taken engineering physics-mechanics, engineering physics-E\&M, and statics. 
Table 7: Five-Year Before and After Averages for Dynamics

\begin{tabular}{|c|c|c|c|c|}
\hline Years & N & Ave GPA & Pass Rate & \% “B” or higher \\
\hline $2006-2010$ & 117 & 2.80 & 89.8 & 53.7 \\
\hline $2011-2016$ & 163 & 3.00 & 90.6 & 64.9 \\
\hline
\end{tabular}

As with the other two courses, student performance in the dynamics class improved after the physics-mechanics class was restructured.

\subsection{Conclusions}

An engineering physics-mechanics class was completely restructured, blending the time normally allocated for separate lecture, laboratory and recitation components into three 2.5 hour classes. Traditional lecture and laboratory elements were replaced with active learning techniques. Assessment of the new course shows significant gains in conceptual understanding, as measured by the FCI test. Grade data also shows improvements in the overall course GPA, the pass rate, and the grade distribution.

Tracking students in three follow-on courses (engineering physics - E\&M, statics, and dynamics), shows that students who took the physics - mechanics class in the new format performed better in those subsequent courses. Improvement was judged based on grade data for the subsequent classes.

In tracking these results, several important observations were noted. First, while there is a great deal of literature on assessing individual courses, very little has been done to track how a course taken early in a student's career impacts later courses. In the present study, there was really no way to assess the impacts beyond using grade data.

The use of grade data for assessing improvement is certainly problematic. For the subject study, data was compiled over a ten-year period. During that time, various instructors taught the courses and it is certainly probable that the grading standards were not consistent. Likewise, even if the instructors remained the same, it is likely that grading variations occurred due to natural differences in the difficulty level of the exams and assignments. It is also possible that the "caliber" of the students may have changed. (However, it is important to note that over the period from 2008 to 2014 the FCI pre-test score remained nearly constant - see Table 1 . This suggests that the students' conceptual understanding of physics entering the class did not change over that time.) Another complicating factor is that the students in the four classes are not the same. As noted earlier, the physics-mechanics and physics - E\&M courses consists of all engineering majors along with engineering management majors and, occasionally, math and/or secondary education majors. However, the statics class contains just mechanical engineering and engineering management majors and the dynamics class is just mechanical engineering majors. Given the data available, it would be possible to extract just the students who have taken all 4 courses and track their progress. However, such an analysis was not done for this paper. Lastly, since all faculty work to improve their classes, it is possible that other courses contributed to the improved performance in the subsequent classes. 
The bottom line is that using grade data to track downstream impacts is questionable and it is very difficult to make concrete conclusions. That said, student performance in all of the related downstream courses did increase after the engineering physics - mechanics course was restructured, which is certainly better than seeing no change or reduced performance. It would certainly be beneficial to have a better method for tracking the improvements in down-stream courses, since our goal as instructors should be to make changes to our courses that have lasting impact.

\section{References}

1. Halloun, I.A., and Hestenes, D., "The initial knowledge state of college physics students," Am. J. Phys. 53, 1056-1055, (1985).

2. Halloun, IA, \& Hestenes, D., "Common-sense concepts about motion," Am. J. Phys. 53, 1056-1065, (1985).

3. Tobias, S., Revitalizing Undergraduate Science; Why Some Things Work and Most Don't, Tucson, AZ: Research Corporation, (1992).

4. Tobias, S., They're Not Dumb, They're Different: Stalking the Second Tier, Tucson, AZ: Research Corporation, (1990).

5. Laws, P., "Calculus-based physics without lectures," Phys. Today 44(12), 24-31, (1991)

6. Laws, P., Workshop Physics, J. Wiley, (2004)

7. Mazur, E., Peer Instruction: A User's Manual, Prentice Hall, (1997).

8. Sokoloff, D. \& Thornton, R., Interactive Lecture Demonstrations, J. Wiley, (2004).

9. Hestenes, D., Wells, M., \& Swackhamer, G., "Force Concept Inventory," Phys. Teach. 30, 141-158 (1992)

10. “Assessment Information Test Page", NC State University, http://www.ncsu.edu/per/TestInfo.html

11. Redish, E., Teaching Physics with the Physics Suite, J. Wiley, (2003).

12. Knight, R., Five Easy Lessons: Strategies for Successful Physics Teaching, Addison Wesley, (2004).

13. Garrison, T., Exploratory Physics: An Active Approach to Learning Physics, 2014 Version, currently self-published, (2014).

14. Garrison, T, "Student Performance Enhancements via an Active, Integrated Engineering Physics Course," Proceedings of $121^{\text {st }}$ ASEE Annual Conference and Exposition, Indianapolis, IN, June 2014.

15. Garrison, T, "Active Learning Laboratories in a Restructured Engineering Physics Mechanics," Proceedings of $122^{\text {st }}$ ASEE Annual Conference and Exposition, Seattle, WA, June 2015.

16. Hake, R., "A six thousand student study of mechanics test data for introductory physics courses,” Am. J. Phys. 66(1), 64-74, (1998). 